\title{
Application of the shooting method to second-order multi-point integral boundary-value problems
}

Huilan Wang ${ }^{*}$, Zigen Ouyang and Liguang Wang

${ }^{*}$ Correspondence:

huilan07@163.com

School of Mathematics and Physics,

University of South China,

Hengyang, 421001, P.R. China

\section{Abstract}

In this paper, we focus on the following second-order multi-point integral boundary-value problem:

$$
\begin{aligned}
& u^{\prime \prime}(t)+a(t) f(u(t))=0, \quad 0<t<1, \\
& u(0)=0, \quad u(1)=\sum_{i=1}^{n} \alpha_{i} \int_{0}^{\eta_{i}} u(s) d s,
\end{aligned}
$$

where $0<\eta_{1}<\eta_{2}<\cdots<\eta_{n}<1, \alpha_{i} \geq 0$ for $i=1, \ldots, n-1$ and $\alpha_{n}>0$ are given constants. The proof is based on the shooting method. By constructing a quadratic function and a sine function as the shooting objects and combining the integral mean value theorem with the comparison principle, we consider the existence of positive solutions to the BVP respectively under the case $0<\sum_{i=1}^{n} \alpha_{i} \eta_{i} \leq 1$ and the case $\sum_{i=1}^{n} \alpha_{i} \eta_{i}>1$. The method is concise and some new criteria are established. MSC: 34B10; 34B15; 34B18

Keywords: shooting method; integral boundary-value problem; positive solution

\section{Introduction}

For the study of nonlinear second-order multi-point boundary-value problem, many results have been obtained by using all kinds of fixed point theorems related to a completely continuous map defined in a Banach space. We refer the reader to [1-9] and the references therein. Some of the results are so classical that little work can exceed; however, most of these papers are concerned with problems with boundary conditions of restrictions either on the slope of solutions and the solutions themselves, or on the number of boundary points $[2,5-8,10]$.

In [8], Ma investigated the existence of positive solutions of the nonlinear second-order m-point boundary value problem

$$
\begin{aligned}
& u^{\prime \prime}(t)+a(t) f(u(t))=0, \quad 0<t<1, \\
& u(0)=0, \quad \sum_{i=1}^{m-2} \alpha_{i} u_{i}\left(\eta_{i}\right)=u(1),
\end{aligned}
$$
dium, provided the original work is properly cited. 
where $0<\eta_{1}<\eta_{2}<\cdots<\eta_{m-2}<1, \alpha_{i} \geq 0$ for $i=1, \ldots, m-3, \alpha_{m-2}>0, f \in C([0, \infty) ;[0, \infty))$, $a \in C([0,1] ;[0, \infty))$, and there exists a $t_{0} \in\left[\eta_{m-2}, 1\right]$ such that $a\left(t_{0}\right)>0$.

Set

$$
f_{0}=\lim _{u \rightarrow 0^{+}} \frac{f(u)}{u}, \quad f_{\infty}=\lim _{u \rightarrow \infty} \frac{f(u)}{u} .
$$

The author obtained the existence of a positive solution to (1.1)-(1.2) under the case $f_{0}=0$ and $f_{\infty}=\infty$ (super-linear case) or the case $f_{0}=\infty$ and $f_{\infty}=0$ (sub-linear case) when $0<$ $\sum_{i=1}^{m-2} \alpha_{i} \eta_{i}<1$.

Recently, Tariboon [9] considered three-point boundary-value problem (1.1) with the integral boundary condition

$$
u(0)=0, \quad u(1)=\alpha \int_{0}^{\eta} u(s) d s
$$

where $0<\eta<1, \alpha>0$.

Such a boundary condition might be more realistic in the mathematical models of thermal conductivity, groundwater flow, thermoelectric flexibility and plasma physics, because it describes the fluid properties in a certain continuous medium. Under the assumption that $0<\alpha \eta^{2}<2$, Tariboon and the author proved that problem (1.1)-(1.3) has at least one positive solution in the super-linear case or in the sub-linear one.

However, the method used in the previous two papers is Krasnoselskii's fixed point theorem in a cone, which relates to constructing a completely continuous cone map in a Banach space, and the proof is somewhat procedural.

Constructively, Agarwal [11] explored the solution of multi-point boundary value problems by converting BVPs to equivalent IVPs, which is called shooting method. After that Man Kam Kwong $[4,12]$ used the shooting method to consider second-order multi-point boundary value problems. In [12], Kwong studied the existence of a positive solution to the following three-point boundary value problem:

$$
\begin{aligned}
& u^{\prime \prime}(t)+f(u(t))=0, \quad 0<t<1, \\
& u(0)=0, \quad \mu u\left(\frac{1}{2}\right)=u(1) .
\end{aligned}
$$

The principle of the shooting method used in [12] is converting BVP (1.4)-(1.5) into finding suitable initial slopes $m>0$ such that the solution of equation (1.4) with the initial value condition

$$
u(0)=0, \quad u^{\prime}(0)=m
$$

vanishes for the first time after $t>1$. Denote by $u(t, m)$ the solution of (1.4)-(1.6) provided it exists. Then solving the boundary value problem is equivalent to finding $m$ such that

$$
\mu u\left(\frac{1}{2}, m\right)=u(1, m)
$$


If we can find two solutions $u\left(t, m_{1}\right)$ and $u\left(t, m_{2}\right)$ of (1.4) such that

$$
u\left(1, m_{1}\right) \geq(\text { or } \leq) \mu u\left(\frac{1}{2}, m_{1}\right)
$$

and

$$
u\left(1, m_{2}\right) \leq(\text { or } \geq) \mu u\left(\frac{1}{2}, m_{2}\right)
$$

where $u\left(t, m_{1}\right)>0, u\left(t, m_{2}\right)>0$ for $t \in(0,1)$, then there must exist a number $m$ between $m_{1}$ and $m_{2}$ such that $u(t, m)$ is the solution of (1.4)-(1.5). By constructing two sine functions as the shooting objects and combining with the comparison principle, the author obtained some better results than those via fixed point techniques for the existence of positive solutions to (1.4)-(1.5).

In this paper, we try to employ the shooting method to establish the existence results of positive solutions for (1.1) with the more generalized multi-point integral boundary condition

$$
u(0)=0, \quad \sum_{i=1}^{n} \alpha_{i} \int_{0}^{\eta_{i}} u(s) d s=u(1)
$$

where $0<\eta_{1}<\eta_{2}<\cdots<\eta_{n}<1, \alpha_{i} \geq 0$ for $i=1, \ldots, n-1$ and $\alpha_{n}>0$ are given constants. Following the principle of the shooting method, there are two obstacles we encounter. The first one is that the boundary condition involves integral from 0 to $\eta_{i}(i=1, \ldots, n)$, so we transform the integral problem into a single-point problem by using the integral mean value theorem. The other difficulty is that we cannot obtain the existence results by constructing two sine functions as in [12] because of the particularity of $\eta=\frac{1}{2}$ in [12]. Therefore, we construct a quadratic function and a sine function as the objective ones.

The purpose of this article lies in two aspects. One is to explore the application of the shooting method in a more complicated multi-point integral boundary value problem, which demonstrates another way in studying BVPs. The other one is to establish new criteria for the existence of positive solutions to (1.1)-(1.7) under the case $0<\sum_{i=1}^{n} \alpha_{i} \eta_{i} \leq 1$ and the case $\sum_{i=1}^{n} \alpha_{i} \eta_{i}>1$.

For the sake of convenience, we denote

$$
\begin{array}{ll}
\max _{0 \leq t \leq 1}\{a(t)\}=a^{L}, & \min _{0 \leq t \leq 1}\{a(t)\}=a^{l}, \\
\bar{f}_{x}=\lim _{u \rightarrow x} \sup \frac{f(u)}{u}, & f_{-x}=\liminf _{u \rightarrow x} \frac{f(u)}{u}, \quad x \in\{0,+\infty\} .
\end{array}
$$

Let $u(t, m)$ be the solution of (1.1)-(1.6) and define

$$
k(m)=\frac{\sum_{i=1}^{n} \alpha_{i} \int_{0}^{\eta_{i}} u(s, m) d s}{u(1, m)} .
$$

In this paper, we always assume:

$\left(\mathrm{H}_{1}\right) f \in C([0, \infty) ;[0, \infty)), a \in C([0,1] ;[0, \infty)), a^{l}>0$. 
Under the assumption, it is not difficult to prove that the initial problem (1.1)-(1.6) has at least one solution defined on $[0,1] \times[0,+\infty)$. In fact, after translating second-order differential equation (1.1) into one-order equations, one can draw the conclusion [13].

Further, we introduce the comparison results derived from $[4,12]$, which evolved from the Sturm comparison theorem.

Theorem 1.1 Let $u(t, m), z(t, m), Z(t, m)$ be the solution of the initial value problems, respectively,

$$
\begin{aligned}
& u^{\prime \prime}(t)+F(u(t))=0, \quad u(0)=0, \quad u^{\prime}(0)=m, \\
& Z^{\prime \prime}(t)+G(Z(t))=0, \quad Z(0)=0, \quad Z^{\prime}(0)=m, \\
& z^{\prime \prime}(t)+g(z(t))=0, \quad z(0)=0, \quad z^{\prime}(0)=m,
\end{aligned}
$$

and suppose that $F, G, g$ are nonnegative continuous functions on a certain interval I for $t \in[0,1]$ and such that

$$
g(\omega) \leq F(\omega) \leq G(\omega), \quad \omega \in I .
$$

If $Z(t)$ does not vanish in $[0,1]$, then for $0<\eta<1$, it yields

$$
\frac{z(\eta)}{z(1)} \leq \frac{u(\eta)}{u(1)} \leq \frac{Z(\eta)}{Z(1)}
$$

The paper is arranged as follows. In the next section, we put forward the basic principle of the shooting method used in this paper, and show that BVP (1.1)-(1.7) has no positive solution when $\sum_{i=1}^{n} \alpha_{i} \eta_{i}^{2}>2$. In Section 3, the general criteria are established for the existence of positive solutions to (1.1)-(1.7) under the case $0<\sum_{i=1}^{n} \alpha_{i} \eta_{i}^{2}<2$. Moreover, we present the special results in the form of corollaries corresponding to the super-linear case or the sub-linear case. Finally, we come to the conclusion and an example is presented to illustrate our results.

\section{Preliminaries}

Lemma 2.1 If there exist two initial slopes $m_{1}>0$ and $m_{2}>0$ such that

(i) the solution $u\left(t, m_{1}\right)$ of (1.1)-(1.6) remains positive in $(0,1)$ and $k\left(m_{1}\right) \leq 1$;

(ii) the solution $u\left(t, m_{2}\right)$ of (1.1)-(1.6) satisfies $u\left(t, m_{2}\right)>0$ for $t \in(0,1)$ and $k\left(m_{2}\right) \geq 1$; then multi-point boundary value problem (1.1)-(1.7) has a positive solution with the slope $u^{\prime}(0)=m_{0}$ between $m_{1}$ and $m_{2}$.

Proof Since the solutions of (1.1)-(1.6) depend on the initial value continuously, then from (1.8), it implies that $k(m)$ is continuous on $m$. In view of the intermediate value theorem of continuous functions, there exists a number $m_{0}$ between $m_{1}$ and $m_{2}$ such that $k\left(m_{0}\right)=1$, that is,

$$
u\left(t, m_{0}\right)=\sum_{i=1}^{n} \alpha_{i} \int_{0}^{\eta_{i}} u\left(s, m_{0}\right) d s
$$

Therefore, $u\left(t, m_{0}\right)$ is the solution of (1.1)-(1.7). 
Lemma 2.2 Let $\sum_{i=1}^{n} \alpha_{i} \eta_{i}^{2}>2$, then (1.1)-(1.7) has no positive solution.

Proof Assume that (1.1)-(1.7) has a positive solution $u$.

If $u(1)>0$, then $\sum_{i=1}^{n} \alpha_{i} \int_{0}^{\eta_{i}} u(s) d s>0$, the convexity of $u$ implies that $u\left(\eta_{i}\right)>0(i=$ $1,2, \ldots, n)$ and

$$
\begin{aligned}
\frac{u(1)}{1} & =\sum_{i=1}^{n} \alpha_{i} \int_{0}^{\eta_{i}} u(s) d s \geq \frac{1}{2} \sum_{i=1}^{n} \alpha_{i} \eta_{i} u\left(\eta_{i}\right) \\
& =\frac{1}{2} \sum_{i=1}^{n} \alpha_{i} \eta_{i}^{2} \frac{u\left(\eta_{i}\right)}{\eta_{i}} \geq \frac{1}{2} \sum_{i=1}^{n} \alpha_{i} \eta_{i}^{2} \frac{u\left(\eta_{n}\right)}{\eta_{n}} \\
& >\frac{u\left(\eta_{n}\right)}{\eta_{n}},
\end{aligned}
$$

which contradicts with the convexity of $u$.

If $u(1)=0$, then $\sum_{i=1}^{n} \alpha_{i} \int_{0}^{\eta_{i}} u(s) d s=0$, that is, $u(t) \equiv 0$ for $t \in\left[0, \eta_{n}\right]$. If there exists $\tau \in$ $\left(\eta_{n}, 1\right)$ such that $u(\tau)>0$, then $u(0)=u\left(\eta_{n}\right)=0$ and $u(\tau)>0$, which contradicts with the convexity of $u$. Therefore $u(t) \equiv 0$ for $t \in[0,1]$.

In the rest of this paper, we always assume:

$\left(\mathrm{H}_{2}\right) \quad 0<\sum_{i=1}^{n} \alpha_{i} \eta_{i}^{2}<2$.

\section{Main results}

Theorem 3.1 Assume that $\left(\mathrm{H}_{1}\right)-\left(\mathrm{H}_{2}\right)$ holds. Suppose $0<\sum_{i=1}^{n} \alpha_{i} \eta_{i} \leq 1$ and there exists a constant $A \in\left[0, \frac{\pi}{2}\right]$ such that

(i) $\bar{f}_{0}<\frac{A^{2}}{a^{L}} \leq \frac{A^{2}}{a^{l}}<f_{-\infty}$; or

(ii) $\bar{f}_{\infty}<\frac{A^{2}}{a^{L}} \leq \frac{A^{2}}{a^{l}}<f_{0}$.

Then problem (1.1)-(1.7) has a positive solution.

Proof (i) Since $\bar{f}_{0}<\frac{A^{2}}{a^{L}}$, we can choose a positive number $m^{*}$ such that

$$
\frac{f(u)}{u} \leq \frac{A^{2}}{a^{L}}, \quad 0<u \leq m^{*} .
$$

We claim that there exists a positive number $m_{1}$ small enough such that $0<u\left(t, m_{1}\right) \leq$ $m_{1} t \leq m_{1}<m^{*}$ for $t \in[0,1]$. The claim is based on the convexity of the function $u\left(t, m_{1}\right)$ and the Sturm comparison theorem (see [12]). Hence,

$$
a(t) f\left(u\left(t, m_{1}\right)\right) \leq a^{L} \frac{A^{2}}{a^{L}} u\left(t, m_{1}\right)=A^{2} u\left(t, m_{1}\right), \quad t \in[0,1] .
$$

Let

$$
Z(t)=\sin A t, \quad t \in[0,1],
$$

then

$$
Z^{\prime \prime}(t)+A^{2} Z(t)=0 .
$$


From (1.8), (3.1) and combining the integral mean value theorem with Theorem 1.1, we have

$$
\begin{aligned}
k\left(m_{1}\right) & =\frac{\sum_{i=1}^{n} \alpha_{i} \int_{0}^{\eta_{i}} u\left(s, m_{1}\right) d s}{u\left(1, m_{1}\right)}=\frac{\sum_{i=1}^{n} \alpha_{i} \eta_{i} u\left(\xi_{i}, m_{1}\right)}{u\left(1, m_{1}\right)} \\
& \leq \frac{u\left(\bar{\xi}, m_{1}\right) \sum_{i=1}^{n} \alpha_{i} \eta_{i}}{u\left(1, m_{1}\right)} \leq \frac{\sin A \bar{\xi} \sum_{i=1}^{n} \alpha_{i} \eta_{i}}{\sin A} \leq \frac{\sin A \eta_{n} \sum_{i=1}^{n} \alpha_{i} \eta_{i}}{\sin A}<1
\end{aligned}
$$

where $\xi_{i} \in\left[\eta_{i-1}, \eta_{i}\right]$ and $\bar{\xi} \in\left\{\xi_{1}, \ldots, \xi_{n}\right\}$ such that $u\left(\bar{\xi}, m_{1}\right)=\max _{1 \leq i \leq n} u\left(\xi_{i}, m_{1}\right)$.

The second inequality in (i) means that there exists a number $M$ large enough such that

$$
\frac{f(u)}{u} \geq \frac{A^{2}}{a^{l}}, \quad u \geq M .
$$

For this $M$, there exist two numbers $\delta$ and $M^{*}$ such that

$$
0<\delta<1-\eta_{n}, \quad M^{*}=\frac{2(1-\delta)-\eta_{n} \sum_{i=1}^{n} \alpha_{i} \eta_{i}^{2}}{2-\sum_{i=1}^{n} \alpha_{i} \eta_{i}^{2}} \times \frac{M A^{2}}{2}
$$

and there exists another number $m_{2} \geq M^{*}$ such that $u\left(t, m_{2}\right) \geq M$ for $t \in[\delta, 1-\delta]$. Set

$$
z(t)= \begin{cases}M^{*} t-\frac{M A^{2}}{2} t^{2}, & t \in[0,1-\delta], \\ M^{*}(1-\delta)-\frac{M A^{2}}{2}(1-\delta)^{2}, & t \in[1-\delta, 1] .\end{cases}
$$

In view of $\left(\mathrm{H}_{2}\right)$ and (3.3), it is not difficult to verify that

$$
M^{*}>\frac{M A^{2}}{2}(1-\delta)
$$

which implies from (3.4) that $z(t)>0$ for $t \in(0,1]$. Thus, by the convexity of $u\left(t, m_{2}\right)$ and Theorem 1.1, we have

$$
\begin{aligned}
k\left(m_{2}\right) & =\frac{\sum_{i=1}^{n} \alpha_{i} \int_{0}^{\eta_{i}} u\left(s, m_{2}\right) d s}{u\left(1, m_{2}\right)} \geq \frac{\sum_{i=1}^{n} \alpha_{i} \eta_{i} u\left(\eta_{i}, m_{2}\right)}{2 u\left(1, m_{2}\right)} \\
& =\frac{\sum_{i=1}^{n} \alpha_{i} \eta_{i}^{2} \frac{u\left(\eta_{i}, m_{2}\right)}{\eta_{i}}}{2 u\left(1, m_{2}\right)} \geq \frac{\sum_{i=1}^{n} \alpha_{i} \eta_{i}^{2} \frac{u\left(\eta_{n}, m_{2}\right)}{\eta_{n}}}{2 u\left(1, m_{2}\right)} \\
& \geq \frac{\sum_{i=1}^{n} \alpha_{i} \eta_{i}^{2} z\left(\eta_{n}\right)}{2 \eta_{n} z(1)}=\frac{\sum_{i=1}^{n} \alpha_{i} \eta_{i}^{2}\left[M^{*}-\frac{M A^{2}}{2} \eta_{n}\right]}{2(1-\delta)\left[M^{*}-\frac{M A^{2}}{2}(1-\delta)\right]} \\
& \geq \frac{\sum_{i=1}^{n} \alpha_{i} \eta_{i}^{2}\left[M^{*}-\frac{M A^{2}}{2} \eta_{n}\right]}{2\left[M^{*}-\frac{M A^{2}}{2}(1-\delta)\right]}=1 .
\end{aligned}
$$

By Lemma 2.1 and (3.2)-(3.5), there exists a number $m_{0}$ between $m_{1}$ and $m_{2}$ such that $u\left(t, m_{0}\right)$ is the positive solution of (1.1)-(1.7). The proof for (i) is complete.

Now, we prove for (ii).

In view of $\bar{f}_{\infty}<\frac{A^{2}}{a^{L}}$, we can choose a number $N$ large enough such that

$$
\frac{f(u)}{u} \leq \frac{A^{2}}{a^{L}}, \quad u \geq N .
$$


For this $N$, there exist a number $\epsilon$ small enough and a number $m_{1}$ large enough such that $0<\epsilon<\eta_{1}$ and $u\left(t, m_{1}\right) \geq N$ for $t \in[\epsilon, 1-\epsilon]$. Therefore

$$
a(t) f\left(u\left(t, m_{1}\right)\right) \leq a^{L} \frac{A^{2}}{a^{L}} u\left(t, m_{1}\right)=A^{2} u\left(t, m_{1}\right), \quad t \in[\epsilon, 1-\epsilon] .
$$

Obviously, $\epsilon \rightarrow 0$ as $m_{1} \rightarrow \infty$. Thus $u\left(t, m_{1}\right) \geq N$ approximately for $t \in[0,1]$ as $m_{1} \rightarrow \infty$.

Let $Z(t)=\sin A t, t \in[0,1]$. Similar to (3.2), we obtain

$$
\begin{aligned}
k\left(m_{1}\right) & =\frac{\sum_{i=1}^{n} \alpha_{i} \int_{0}^{\eta_{i}} u\left(s, m_{1}\right) d s}{u\left(1, m_{1}\right)}=\frac{\sum_{i=1}^{n} \alpha_{i} \eta_{i} u\left(\xi_{i}, m_{1}\right)}{u\left(1, m_{1}\right)} \\
& \leq \frac{u\left(\bar{\xi}, m_{1}\right) \sum_{i=1}^{n} \alpha_{i} \eta_{i}}{u\left(1, m_{1}\right)} \leq \frac{\sin A \bar{\xi} \sum_{i=1}^{n} \alpha_{i} \eta_{i}}{\sin A} \leq \frac{\sin A \eta_{n} \sum_{i=1}^{n} \alpha_{i} \eta_{i}}{\sin A}<1,
\end{aligned}
$$

where $\xi_{i} \in\left[\eta_{i-1}, \eta_{i}\right]$ and $\bar{\xi} \in\left\{\xi_{1}, \ldots, \xi_{n}\right\}$ such that $u\left(\bar{\xi}, m_{1}\right)=\max _{1 \leq i \leq n} u\left(\xi_{i}, m_{1}\right)$.

Since $f_{-0}>\frac{A^{2}}{a^{l}}$, then there exist two positive numbers $m_{2}$ and $\sigma$ small enough such that

$$
\frac{f(u)}{u} \geq \frac{A^{2}}{a^{l}}, \quad \sigma \leq u \leq m_{2} .
$$

By the convexity of $u\left(t, m_{2}\right)$, for these $\sigma$ and $m_{2}$, there exists a positive number $\tau$ small enough such that

$$
0<\tau<\eta_{1}, \quad \sigma \leq u\left(t, m_{2}\right) \leq m_{2}, \quad t \in[\tau, 1]
$$

which yields

$$
a(t) f\left(u\left(t, m_{2}\right)\right) \geq a \frac{a^{l}}{a^{2}} u\left(t, m_{2}\right) \geq A^{2} \sigma, \quad t \in[\tau, 1] .
$$

Let

$$
m^{*}=\frac{2-\eta_{n} \sum_{i=1}^{n} \alpha_{i} \eta_{i}^{2}}{2-\sum_{i=1}^{n} \alpha_{i} \eta_{i}^{2}} \frac{A^{2} \sigma}{2}
$$

and

$$
z(t)=m^{*} t-\frac{A^{2} \sigma}{2} t^{2}, \quad t \in[\tau, 1]
$$

From (3.6) and (3.7), we have $m^{*}>\frac{A^{2} \sigma}{2}$ and $z(t)>0$ for $t \in(0,1]$. Thus

$$
\begin{aligned}
k\left(m_{2}\right) & =\frac{\sum_{i=1}^{n} \alpha_{i} \int_{0}^{\eta_{i}} u\left(s, m_{2}\right) d s}{u\left(1, m_{2}\right)} \geq \frac{\sum_{i=1}^{n} \alpha_{i} \eta_{i} u\left(\eta_{i}, m_{2}\right)}{2 u\left(1, m_{2}\right)} \\
& \geq \frac{\sum_{i=1}^{n} \alpha_{i} \eta_{i}^{2} \frac{u\left(\eta_{i}, m_{2}\right)}{\eta_{i}}}{2 u\left(1, m_{2}\right)} \geq \frac{\sum_{i=1}^{n} \alpha_{i} \eta_{i}^{2} \frac{u\left(\eta_{n}, m_{2}\right)}{\eta_{n}}}{2 u\left(1, m_{2}\right)} \\
& \geq \frac{\sum_{i=1}^{n} \alpha_{i} \eta_{i}^{2} z\left(\eta_{n}\right)}{2 \eta_{n} z(1)} \geq \frac{\sum_{i=1}^{n} \alpha_{i} \eta_{i}^{2}\left[m^{*}-\frac{A^{2} \sigma}{2} \eta_{n}\right]}{2\left[m^{*}-\frac{A^{2} \sigma}{2}\right]}=1 .
\end{aligned}
$$

By Lemma 2.1, the proof for (ii) is complete. 
Theorem 3.2 Assume that $\left(\mathrm{H}_{1}\right)-\left(\mathrm{H}_{2}\right)$ holds. Suppose $\sum_{i=1}^{n} \alpha_{i} \eta_{i}>1$ and there exists a constant $A \in\left[0, \frac{\pi}{2}\right]$ such that

$$
\frac{\sin A}{\sin \eta_{n} A}=\sum_{i=1}^{n} \alpha_{i} \eta_{i} .
$$

Then problem (1.1)-(1.7) has a positive solution under the case

(i) $\bar{f}_{0}<\frac{A^{2}}{a^{L}} \leq \frac{A^{2}}{a^{l}}<f_{-\infty}$; or

(ii) $\bar{f}_{\infty}<\frac{A^{2}}{a^{L}} \leq \frac{A^{2}}{a^{l}}<f_{0}$.

Proof Note the computation of $k\left(m_{1}\right)$ in Theorem 3.1. In (3.2), if we substitute $\sum_{i=1}^{n} \alpha_{i} \eta_{i}$ with

$$
\frac{\sin A}{\sin \eta_{n} A}
$$

then $k\left(m_{1}\right) \leq 1$, and all the steps in the following are the same as in Theorem 3.1.

Now, let us consider the special super-linear case or the sub-linear case. It is not difficult to verify the following corollaries.

Corollary 3.1 Assume that $0<\sum_{i=1}^{n} \alpha_{i} \eta_{i} \leq 1$ and

(i) $f_{0}=0, f_{\infty}=\infty$; or

(ii) $f_{0}=\infty, f_{\infty}=0$.

Then problem (1.1)-(1.7) has a positive solution.

Corollary 3.2 If $\sum_{i=1}^{n} \alpha_{i} \eta_{i}>1$ and there exists a constant $A \in\left[0, \frac{\pi}{2}\right]$ such that

$$
\frac{\sin A}{\sin \eta_{n} A}=\sum_{i=1}^{n} \alpha_{i} \eta_{i} .
$$

Then, problem (1.1)-(1.7) has a positive solution under the case

(i) $f_{0}=0, f_{\infty}=\infty$; or

(ii) $f_{0}=\infty, f_{\infty}=0$.

\section{Conclusion and examples}

The tool which we used for the analysis in this article is the shooting method derived from $[4,12]$; however, we considered a more general problem which involves integral boundaryvalue and multiplicity of boundary-point. The meaningful work that we have done lies in the following three aspects. The first one is that we transform the integral problem into a single-point value one by using the integral mean value theorem. The other one is that we construct a quadratic function and a sine function as the comparison functions because it does not take effect to construct two sine functions as in [12]. Finally, we established the new criteria for the existence of positive solutions to (1.1)-(1.7) under the case $\sum_{i=1}^{n} \alpha_{i} \eta_{i} \leq 1$ and the case $\sum_{i=1}^{n} \alpha_{i} \eta_{i}>1$. Obviously, (1.7) vanishes to (1.3) when $n=1$ and the sup-linear case or the sub-linear case is sufficient for the conditions in Theorem 3.1 and Theorem 3.2, so some of our results are more general or better than those via fixed point techniques. 
Figure 1 Numerical simulation for Example 4.1.

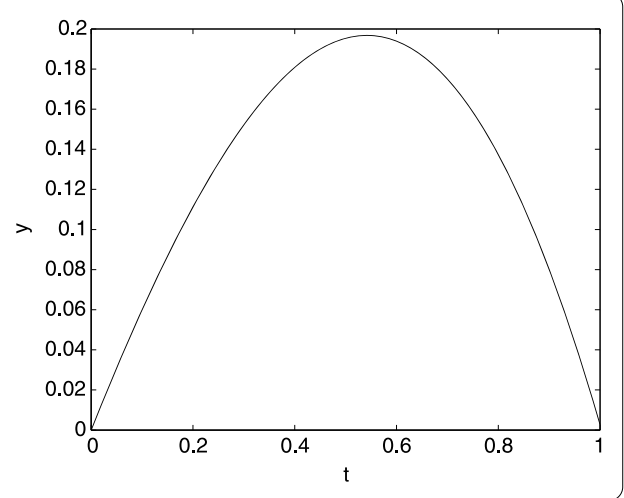

However, in Theorem 3.2, whether the transcendental equation has a solution is somewhat difficult to verify. It can be seen that each method has its pros and cons.

Example 4.1 Consider the BVP

$$
\begin{aligned}
& y^{\prime \prime}(t)+(2 t+1)\left(\frac{2 y}{3}+1\right)=0, \quad 0<t<1, \\
& y(0)=0, \quad y(1)=\frac{1}{4} \int_{0}^{\frac{1}{4}} y(s) d s+\frac{83}{60} \int_{0}^{\frac{3}{4}} y(s) d s,
\end{aligned}
$$

where

$$
a(t)=2 t+1, \quad f(y)=\frac{2 y}{3}+1, \quad \alpha_{1}=\frac{1}{4}, \quad \eta_{1}=\frac{1}{4}, \quad \alpha_{2}=\frac{83}{60}, \quad \eta_{2}=\frac{3}{4} .
$$

It is not difficult to see that

$$
a^{L}=3, \quad a^{l}=1, \quad \bar{f}_{\infty}=\frac{2}{3}, \quad f_{-0}=\infty, \quad \sum_{i=1}^{2} \alpha_{i} \eta_{i}=1.1>1, \quad \sum_{i=1}^{2} \alpha_{i} \eta_{i}^{2}<2 .
$$

In view of $\frac{\sin A}{\sin \frac{3}{4} A}=1.1$, Matlab software gives $A=1.5173$ and $A^{2}=2.3022$. Hence

$$
\bar{f}_{\infty}=\frac{2}{3}<\frac{A^{2}}{a^{L}}<\frac{A^{2}}{a^{l}}<f_{-0}=\infty .
$$

Therefore, the condition (ii) of Theorem 3.2 is satisfied. A numerical simulation (Figure 1) for Example 4.1 demonstrates that BVP (4.1)-(4.2) has a positive solution $y(t)$ such that $y(1)=0.0027$.

The authors declare that they have no competing interests. 


\section{Acknowledgements}

The authors would like to thank the editors and the anonymous referees for their valuable suggestions on the improvement of this paper. First author was partially supported by the Scientific Research Fund of Hunan Provincial Educational Department (1200361), Project of Science and Technology Bureau of Hengyang, Hunan Province (2012KJ2). Second author was partially supported by the Doctor Foundation of University of South China ( No. 5-XQD-2006-9), the Foundation of Science and Technology Department of Hunan Province (No. 2009RS3019), the Natural Science Foundation of Hunan Province (No. 13JJ3074) and the Subject Lead Foundation of University of South China (No. 2007XQD13).

\section{Received: 3 March 2013 Accepted: 8 August 2013 Published: 9 September 2013}

\section{References}

1. Chasreechai, S, Tariboon, J: Positive solutions to generalized second-order three-point boundary value problem. Electron. J. Differ. Equ. 14, 1-14 (2011)

2. Gupta, CP: Solvability of a three-point nonlinear boundary value problem for a second order ordinary differential equations. J. Math. Anal. Appl. 168, 540-551 (1992)

3. Kwong, MK, Wong, JSW: Some remarks on three-point and four-point BVP's for second-order nonlinear differential equations. Electron. J. Qual. Theory Differ. Equ. 20, 1-18 (2009)

4. Kwong, MK, Wong, JSW: The shooting method and nonhomogeneous multipoint BVPs of second-order ODE. Bound. Value Probl. (2007). doi:10.1155/2007/64012.

5. Li, J, Shen, J: Multiple positive solutions for a second-order three-point boundary value problems. Appl. Math. Comput. 182(1), 258-268 (2006)

6. Liu, B, Liu, L, Wu, Y: Positive solutions for singular second order three-point boundary value problems. Nonlinear Anal., Theory Methods Appl. 66, 2756-2766 (2007)

7. Ma, R: Positive solutions for second-order three-point boundary value problems. Comput. Math. Appl. 40, 193-204 (2000)

8. Ma, R: Positive solutions of a nonlinear m-point boundary value problem. Comput. Math. Appl. 42, 755-765 (2001)

9. Tariboon, J, Sitthiwirattham, T: Positive solutions of a nonlinear three-point integral boundary value problem. Bound. Value Probl. (2010). doi:10.1155/2010/519210

10. Kwong, MK, Wong, JSW: Solvability of second-order nonlinear three-point boundary value problems. Nonlinear Anal. 73, 2343-2352 (2010)

11. Agarwal, RP: The numerical solution of multipoint boundary value problems. J. Comput. Appl. Math. 5, 17-24 (1979)

12. Kwong, MK: The shooting method and multiple solutions of two/multi-point BVPS of second-order ODE. Electron. J. Qual. Theory Differ. Equ. 6, 1-14 (2006)

13. You, BL: Supplemental Tutorial of Ordinary Differential Equations. Science Press, Beijing (1987) (in Chinese)

doi:10.1186/1687-2770-2013-205

Cite this article as: Wang et al.: Application of the shooting method to second-order multi-point integral boundary-value problems. Boundary Value Problems 2013 2013:205.

\section{Submit your manuscript to a SpringerOpen ${ }^{\odot}$ journal and benefit from:}

- Convenient online submission

- Rigorous peer review

Immediate publication on acceptance

- Open access: articles freely available online

- High visibility within the field

- Retaining the copyright to your article 